\title{
PRODUCTION OF TERAHERTZ SEED RADIATION FOR FEL/IFEL MICROBUNCHERS FOR SECOND GENERATION PLASMA BEATWAVE EXPERIMENTS AT NEPTUNE
}

\author{
J. E. Ralph, S. Ya. Tochitsky, C. Sung, C. Joshi, J. Rosenzweig \\ University of California at Los Angeles, Los Angeles, California
}

\begin{abstract}
To achieve phase locked injection of short electron bunches in a plasma beatwave accelerator, the Neptune Laboratory will utilize microbunching in an FEL or IFEL system. These systems require terahertz $(\mathrm{THz})$ seed radiation on the order of $10 \mathrm{~kW}$ for the FEL and $10 \mathrm{MW}$ for the IFEL bunchers. We report results of experiments on $\mathrm{THz}$ generation using nonlinear frequency mixing of $\mathrm{CO}_{2}$ laser lines in GaAs. A two-wavelength laser beam was split and sent onto a $2.5 \mathrm{~cm}$ long GaAs crystal cut for noncollinear phase matching. Low power measurements achieved $\sim 1 \mathrm{~W}$ of $340 \mu \mathrm{m}$ radiation using $200 \mathrm{~ns} \mathrm{CO}_{2}$ pump pulses with wavelengths $10.3 \mu \mathrm{m}$ and $10.6 \mu \mathrm{m}$. We also demonstrated tunability of difference frequency radiation, producing $240 \mu \mathrm{m}$ by mixing two different $\mathrm{CO}_{2}$ laser lines. By going to shorter laser pulses and higher intensities, we were able to increase the conversion efficiency while decreasing the surface damage threshold. Using 200ps pulses we produced $\sim 2 \mathrm{MW}$ of $340 \mu \mathrm{m}$ radiation. Future studies in this area will focus on developing large diameter Quasi-Phase matched structures for production of high power $\mathrm{THz}$ radiation using collinear two frequency radiation.
\end{abstract}

\section{INTRODUCTION}

One of the primary goals of the Neptune Laboratory at UCLA is the phase locked injection of relativistic electrons from the Neptune linac into a laser driven relativistic plasma beatwave[1]. Here the electric field of an amplitude modulated high intensity laser pulse generates a space charge wave by longitudinally bunching the plasma. The resultant longitudinal field can be used to accelerate electrons at a very high gradient up to $1 \mathrm{GeV} / \mathrm{m}$ for a plasma density of $10^{16} \mathrm{~cm}^{-1}[2,3]$.

At Neptune, the beat-wave generated by $10.3 \mu \mathrm{m}$ and $10.6 \mu \mathrm{m}$ produces a relativistic plasma wave with a wavelength equal to $343 \mu \mathrm{m}$. The Neptune laser system is currently capable of producing a 200 Joule 200ps laser pulse on two wavelengths [4].

Measurements of accelerated electron spectra from both resonant [3] and non-resonant [5] plasma beatwaves have produced a $100 \%$ energy spread since the electron bunch is much longer than the accelerating region of the plasma wave.

One approach to phase locking microbunches is to drive an inverse free electron laser (IFEL) as a buncher with electromagnetic radiation at the difference frequency of the two laser frequencies [6]. This approach offers very short bunches of electrons in the range of $100 \mathrm{fs}$ that have a direct correlation with the laser beatwave.

Simulations show that this method of microbunching requires a $10 \mathrm{MW}$ pulse of $343 \mu \mathrm{m}$ radiation as the pump for the IFEL [7].

A complimentary approach which is being pursued at Neptune is the use of a free electron laser [7] with similar bunching and synchronization. This approach has the advantage of decreasing the power requirements of the $\mathrm{THz}$ radiation to approximately $10 \mathrm{~kW}$. However the electron beam current needed is in the range of $100 \mathrm{~A}$ for the FEL where as only test particles are needed for the IFEL. Both approaches have the advantage of using the same undulator and require a $\mathrm{THz}$ source.

\section{BACKGROUND}

According to theory, the coupled wave equations for nonlinear optical processes can result in a new source leading to difference frequency generation (DFG) where,

$$
\omega_{1}-\omega_{2}=\omega_{3}
$$

Here $\omega_{1}, \omega_{2}$ and $\omega_{3}$ correspond to $10.3 \mu \mathrm{m}, 10.6 \mu \mathrm{m}$ and $340 \mu \mathrm{m}$ respectively. To maximize the nonlinear effect over a length much longer than the length by which the waves become $\pi / 2$ out of phase, phase matching is required.

$$
\vec{k}_{1}-\vec{k}_{2}-\vec{k}_{3}=\Delta \vec{k}
$$

Phase matching occurs when the phase mismatch $\Delta \vec{k}$ of the wave vectors goes to 0 . Since GaAs is an isotropic crystal, with refractive indices different for $\mathrm{k}_{3}, \mathrm{k}_{2}$ and $\mathrm{k}_{1}$, therefore noncollinear phase matching was required for matching in a bulk crystal.

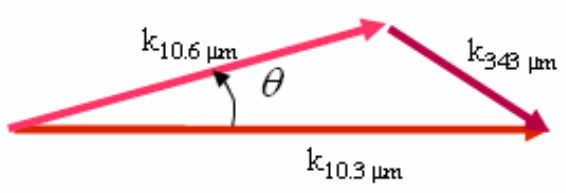

Figure 1: Noncollinear Phase Matching

Generation of $\mathrm{THz}$ radiation by mixing two $\mathrm{CO}_{2}$ laser lines noncollinearly in GaAs was first demonstrated using two $\mathrm{CO}_{2}$ lasers in a cryogenically cooled GaAs sample in experiments at MIT [8]. The anomalous dispersion of the crystal allowed for noncollinear phase-matching. GaAs presents a good choice for frequency mixing due to its high damage threshold, high nonlinear coefficient of 50 $\mathrm{pm} / \mathrm{V}$ as well its availability in large aperture high quality 
crystals. In this paper, we describe the results of our study of generation of $\mathrm{THz}$ radiation using noncollinear phasematching in GaAs at room temperature.

\section{MEASUREMENTS}

\section{Low Power Measurements}

The goal of the low power measurements was to study the phase matching conditions for noncollinear configuration at room temperature in addition to developing a $\mathrm{THz}$ seed radiation source for future FEL microbuncher experiments.

The nonlinear crystal used in all experiments is a semiinsulating large aperture GaAs crystal $(2.0 \mathrm{~cm} \times 4.0 \mathrm{~cm} \times$ $2.5 \mathrm{~cm}$ ) shown below in Figure 2. To decrease $\mathrm{THz}$ absorption, the sample used was Chromium doped which reduces free carrier concentration; the crystal has a specific resistivity greater than $3 \times 10^{8} \mathrm{ohm} \mathrm{cm}$. To maximize the nonlinearity, the crystal was cut so that the polarization of the incident light is parallel to the [111] axis by cutting a surface along (110) plane.

For low power, we used a $1 \mathrm{~Hz}$, two wavelength $\mathrm{CO}_{2}$ $125 \mathrm{kw}$ laser with 250ns FWHM pulses for pumping. The beam was focused to $1 \mathrm{~mm}$ at the crystal.

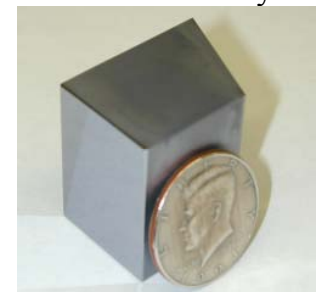

Figure 1: Large aperture GaAs crystal

For noncollinear geometry, the phase matching angle is found according to equation 3 below.

$\theta_{\text {phasematch }}=\cos ^{-1}\left(\frac{k_{1}^{2}+k_{2}^{2}-k_{3}^{2}}{2 k_{1} k_{2}}\right)$

Here the angle is the internal phasematching angle. The external angle is found by applying Snell's law. An external phasematching angle of 2.24 degrees was calculated. We measured a maximum in signal at the angle 2.32 degrees. The wavelength of the newborn radiation was verified with a narrowband filter centered at $350 \mu \mathrm{m}$ with a FWHM of $2 \mathrm{~cm}^{-1}$. All THz signal measurements were made using a Golay cell. This measurement produced 2 watts of difference frequency radiation at $343 \mu \mathrm{m}$ in a $250 \mathrm{~ns}$ pulse. Additional measurements mixing a different pair of lines produced newborn radiation at $240 \mu \mathrm{m}$ with roughly the same efficiency at room temperature.

Measurements of the profile were taken $50 \mathrm{~mm}$ from the back surface of the GaAs crystal using a Golay cell mounted on two translation stages and fitted with a 2.5 $\mathrm{mm}$ iris. The beam profile of the newborn radiation is shown in figure 2. The profile of the beam was elliptical as can be expected from noncollinear mixing.

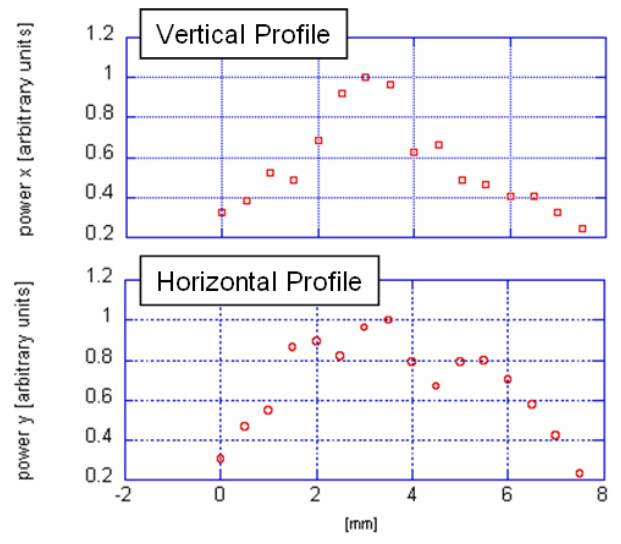

Figure 2: Beam profile measurements of the $\mathrm{THz}$ beam

Figure 3 is a plot of the angle scan measurements. The width of the angle scan corresponds to an effective interaction length between $8 \mathrm{~mm}$ and $10 \mathrm{~mm}$ as shown. Note that for noncollinear configuration, the interaction lenth is limited by the length over which the beams are overlapped. The full width half maximum of the angle is 0.12 degrees. The optical setup used has an angle error of 0.011 degrees.

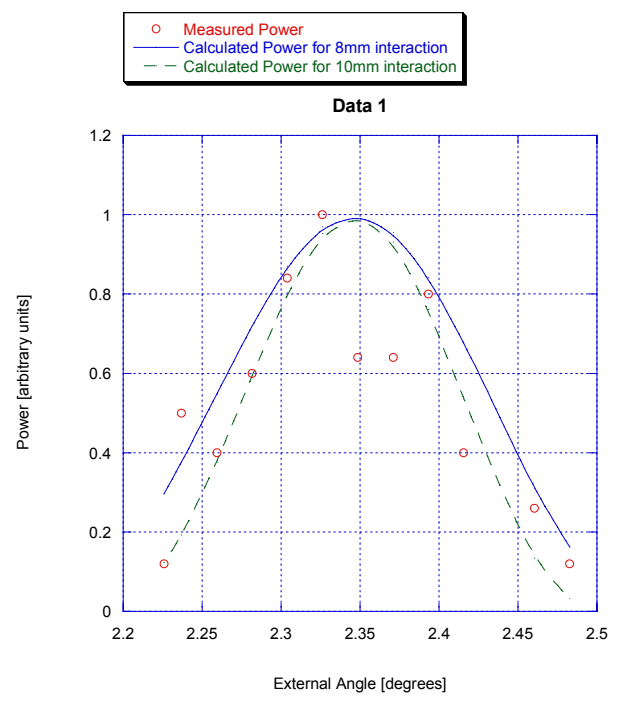

Figure 3: Angle Scan Data vs. Calculations

The newborn beam was coupled to a $5 \mathrm{~mm}$ radius copper waveguide by through a horn. No attenuation losses were measured over a length of 1 meter. Total losses were dominated by the coupling efficiency of the horn. This demonstrates the feasability of guiding over an extended distance which is required for the meter or longer FEL undulator.

\section{High Power Measurements}

The goal of high power measurements was to demonstrate the feasibility of generating high power $\mathrm{THz}$ 
radiation which can be used to seed an IFEL microbuncher.

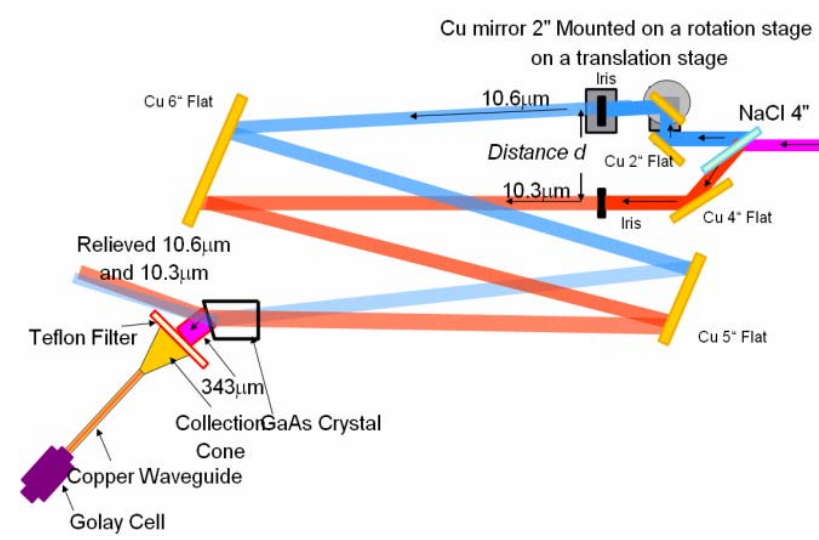

Figure 4: Schematic of High Power Optical Setup

The optical setup shown in figure 4 used for these measurements was designed to provide greater than 0.01 degree angle accuracy and is similar to the setup used for low power measurements. The two wavelength pulse was split using a 60 degree $\mathrm{NaCl}$ beam splitter since at high power using a grating is difficult due to damage threshold. By varying the distance $d$ between the two irises, very good precision was achieved. Since the angle $\theta$ is equal to $\tan ^{-1}(\mathrm{~d} /$ pathlength), having a pathlength longer than 3 $\mathrm{m}$ resulted in very good precision. Additionally, to achieve maximum interaction between the short pulses, the path length difference between the arms was adjusted to less than $5 \mathrm{~mm}$.

The Neptune laser system can generate high power $\mathrm{THz}$ radiation owing to three main factors. First, the Neptune $\mathrm{CO}_{2}$ laser produces short pulses on the order of $200 \mathrm{ps}$. Measurements performed at Neptune demonstrated a surface damage threshold for GaAs of $30 \mathrm{MW} / \mathrm{cm}^{2}$ for long $200 \mathrm{~ns}$ pulses. However by going to 200ps pulses, the damage threshold increased to roughly $2 \mathrm{GW} / \mathrm{cm}^{2}$.

Second, the laser system produces a beam up to 6 inches in diameter. This entire beam can be used for difference frequency generation. Third, due to the fact that very large high quality GaAs crystals are readily available from the semiconductor industry, the ability to produce very high power $\mathrm{THz}$ radiation is a matter of scaling. Limitations of power are imposed only by the damage threshold of GaAs. Power scaling is governed by the equation 4 assuming all beams are the same size.

$$
P_{344}=\frac{\omega_{3}\left|d_{e f f}\right|^{2}}{c^{3}{ }_{0} n_{1} n_{2} n_{3}} P_{10.60} P_{10.28} l_{e f f}^{2}
$$

This equation assumes no absorption and perfect phase matching. Using the crystal described above, we measured $2 \mathrm{MW}$ of $\mathrm{THz}$ radiation which agrees within a factor of 2 with calculations after accounting for Fresnel losses on all surfaces and $6 \mathrm{~cm}^{2}$ pump. Each arm contained both $10.6 \mu \mathrm{m}$ and $10.3 \mu \mathrm{m}$ components, however only one wavelength in each arms was used for the DFG process of interest. The pump beam was a collimated beam as summarized in Table 1 below. We see an increase in conversion efficiency as is implied by equation 2 due to the increase in pump intensity.

Table 1: Pump Specifications

\begin{tabular}{|c|c|c|c|c|}
\hline $\begin{array}{c}\text { Pulse } \\
\text { Repetition } \\
\text { rate }\end{array}$ & Intensity & $\begin{array}{c}\text { Pulse } \\
\text { Length }\end{array}$ & Spot Size & $\begin{array}{c}\mathrm{THz} \\
\text { Power }\end{array}$ \\
\hline $1 \mathrm{~Hz}$ & $5 \mathrm{~mW}$ & $200 \mathrm{~ns}$ & $1 \mathrm{~mm}$ & $2 \mathrm{~W}$ \\
\hline $\begin{array}{c}1 \text { shot } / 5 \\
\text { minutes }\end{array}$ & $\begin{array}{c}\sim \mathrm{GW} / \mathrm{c} \\
\mathrm{m}^{2}\end{array}$ & $250 \mathrm{ps}$ & $\begin{array}{c}20 \mathrm{~mm} \times \\
30 \mathrm{~mm}\end{array}$ & $2 \mathrm{MW}$ \\
\hline
\end{tabular}

For the beam size we had, the effective interaction length of the two beams was the full length of the crystal. Extending this length has the effect of increasing conversion efficiency, however it decreases the angle tolerances for noncollinear geometry. The full width half maximum of the angle scan for high power is only .06 degrees. By going to a large aperture crystal of shorter length, similar high power can be achieved with about the same tolerance as in the low power case.

\section{CONCLUSIONS AND FUTURE PLANS FOR THZ GENERATION AT NEPTUNE}

The measurements outlined above clearly show the potential for achieving the $\mathrm{THz}$ power requirements of both the IFEL and FEL bunching schemes. By increasing the pump and increasing the effective length, a $1 \mathrm{~Hz} 10$ $\mathrm{kW}$ FEL seed source will be possible. One method for increasing the interaction length in the crystal for both low power and high power is to use a quasi-phase matched structure which will allow collinear phase matching over an extended length. This possibility is being explored.

Work supported by the U.S. Department of Energy under Contract No. DE-FG03-92ER40727.

\section{REFERENCES}

[1] S. Ya Tochitsky, P. Musumecci, C. E. Clayton, C. Pellegrini, J. B. Rozenzweig and C. Joshi, Advances Accelerator Concepts 2002, AIP Conference Proceedings 647, Melville, New York, pp.786-795

[2] C. Joshi et. al., Nature, 311, p.529(1984)

[3] S. Ya. Tochitsky et. al., Phys. Rev. Lett. 92, 9(2004)

[4] S. Ya. Tochitsky et. al., Opt. Lett. 24,1717(1999)

[5] C. V. Filip et. al. , Phys. Rev. E. 69, (2004)

[6] P. Musumecci et. al., Proceedings of the International Conference on Lasers 2001, edited by V. J. Corcoran, STS Press, McLean, VA, 2002, pp.41-48

[7] C. Sung et. al., (This Proceedings)

[8] R. L. Aggraval, B. Lax and G. Favrot, Appl. Phys. Lett., Vol. 22, No 7, 1973 